\title{
MicroRNA expression profiling on arrays enhanced with locked nucleic acids
}

\author{
Here we describe a new array system (miRCURY'⿳一 LNA Array) for global microRNA (miRNA) profiling \\ of biological samples. The miRCURY LNA Array system uses locked nucleic acid (LNA $\left.{ }^{T M}\right)$, a high-affinity \\ nucleotide analog, to enhance the array capture probes, giving them high sensitivity and excellent \\ selectivity for miRNA targets. We have simultaneously developed a straightforward method for \\ labeling miRNAs in a sample. As a result, the miRCURY LNA Array system can provide sensitive \\ and accurate profiling of miRNAs while requiring less sample and keeping sample handling steps \\ to a minimum.
}

Arrays present an obvious research tool for determining global miRNA expression profiles. Thus, several commercial and noncommercial platforms for miRNA profiling are currently available, most of which use DNA capture probes. But for short RNA targets, in the range of 18-24 nucleotides, DNA-based capture and detection has substantial limitations. Very recently, LNAs have been shown to be an extremely useful tool for miRNA research, permitting efficient in situ detection and knockdown of miRNAs. Many of the limitations of DNA probes for the detection of short nucleotide targets are known to be overcome by using LNA-based probes ${ }^{1}$. Hence, we set out to develop an array for miRNA profiling based on LNA (Fig. 1), to overcome the limitations of DNA capture probes.

\section{Locked nucleic acid}

LNA is a bicyclic high-affinity RNA mimic in which the sugar ring is locked in the $3^{\prime}$ endo conformation by the introduction of a methylene bridge group connecting the $2^{\prime}-\mathrm{O}$ atom with the $4^{\prime}-\mathrm{C}$ atom. It has been regularly demonstrated that the incorporation of LNA into an oligonucleotide greatly increases the affinity of that oligonucleotide for its complementary target. This is expressed as an increase in melting temperature $\left(T_{\mathrm{m}}\right)$, which increases by $2-8{ }^{\circ} \mathrm{C}$ per LNA base incorporated. In addition, the $T_{\mathrm{m}}$ difference between a perfectly matched target and a mismatched target is substantially higher than that observed when a DNA-based oligonucleotide is used. The combination of higher $T_{\mathrm{m}}$ with excellent mismatch discrimination ability means that LNAbased probes are excellent tools for short targets like miRNA, where

\section{Peter Roberts}

Exiqon A/S, Bygstubben 9, DK-2950, Vedbæk, Denmark. Correspondence should be addressed to P.R. (ptr@exiqon.com).

PUBLISHED ONLINE 22 MARCH 2006; DOI:10.1038/NMETH869

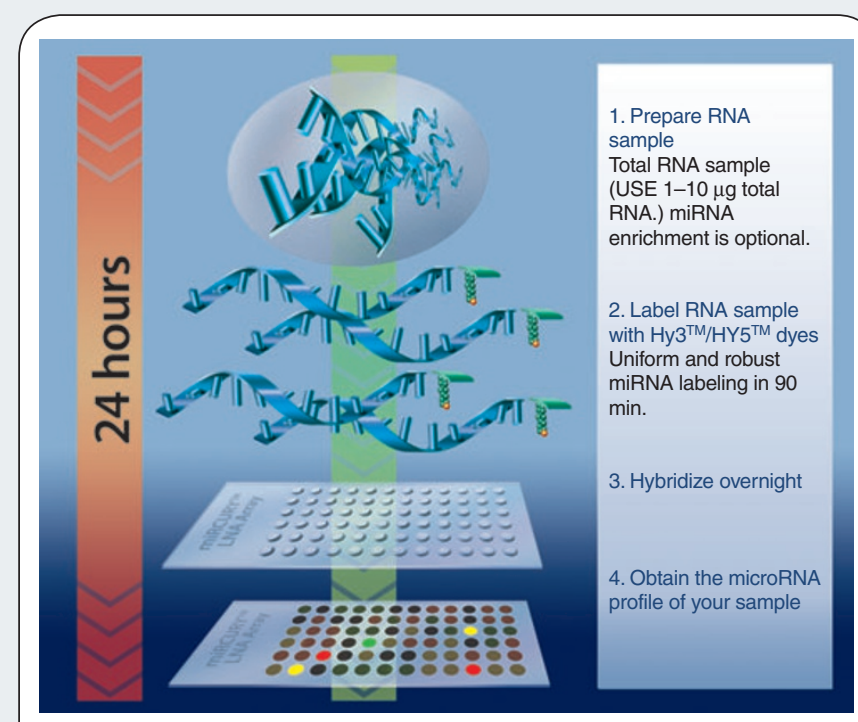

Figure $\mathbf{1} \mid$ The basic steps in using miRCURY Array for miRNA profiling.

the length of the target is between 18 and 24 nucleotides and related miRNAs may only differ from each other by a single base.

\section{Using LNA to overcome the limitations of DNA-based} array capture for miRNAs

There are several issues to consider when designing array methods for miRNAs. The most obvious of these is that the target is short-less than 25 nucleotides - and so the choice of target sequence is extremely limited. This has a strong influence on the design of capture probes. For example, the calculated $T_{\mathrm{m}}$ of miRNAs toward complementary DNA strands can range from 45 to $74{ }^{\circ} \mathrm{C}$. This makes it difficult to design array capture probes based on DNA that will hybridize with equal affinity to all targets at a given hybridization temperature. In comparison, a full-length LNA-enhanced oligonucleotide probe for LNA can 


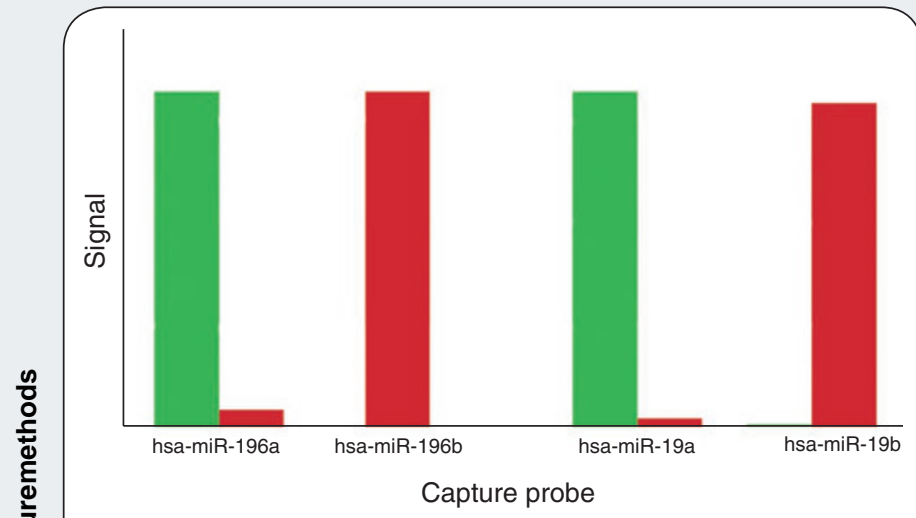

Figure $2 \mid$ miRCURY LNA Arrays are highly specific for their miRNA targets. Two pools of synthetic miRNAs ( $2 \mathrm{fmol}$ of each miRNA) were spiked into a complex background of yeast total RNA $(1 \mu \mathrm{g} / \mu \mathrm{l})$ with hsa-mir-196a/hsa-mir-19a in one pool and hsa-mir-196b/hsa-mir-19b in the other pool. The $a$ and $b$ forms of has-mir-19 and has-mir-196 differ from each other by a single nucleotide. One pool was labeled with $\mathrm{Hy}_{3}{ }^{\mathrm{TM}}$ and the other pool was labeled with $\mathrm{Hy} 5^{\mathrm{TM}}$ using the miRCURY LNA Array Labeling Kit. The reactions were pooled and hybridized onto miRCURY LNA Arrays. The perfect-match:mismatch ratios are in the range of $32-1,110$.

easily have a $T_{m}$ of $80^{\circ} \mathrm{C}$. By varying the length and LNA content of the capture probes in our miRCURY LNA Arrays, we were able to design a set of capture probes $T_{\mathrm{m}}$-normalized to $72{ }^{\circ} \mathrm{C}$ in order to ensure that hybridization efficiency is equal under any particular condition.

Another well-known aspect of miRNAs is that many are closely related to each other (the let-7 family being the most well-known example of this). Some miRNAs differ from each other by only a single nucleotide. LNA is already known for its enhanced ability to discriminate between single mismatches, for example in single-nucleotide polymorphism analysis. This is due to a larger difference in $T_{\mathrm{m}}$ (that is, $\Delta T_{\mathrm{m}}$ ) when a mismatch occurs, compared with that in DNA-based probes. In combination with the conditions of high hybridization stringency that are possible with LNA-based probes, the miRCURY LNA Array platform thus offers an excellent basis for discriminating between even closely related miRNA targets. This has indeed been shown to be the case for some closely related miRNAs (Fig. 2). Future versions of the miRCURY LNA Arrays will be designed to further enhance this feature.

The relatively low sensitivity of DNA-based arrays when it comes to detection of miRNA in samples of total RNA has necessitated the development of techniques for improved sample preparation and signal amplification. Methods for making 'micro cDNA' from miRNA, in a manner analogous to the preparation of cDNA from RNA targets, are difficult and require multiple steps. Hence, the preferred methods for profiling miRNA have favored a miRNA enrichment

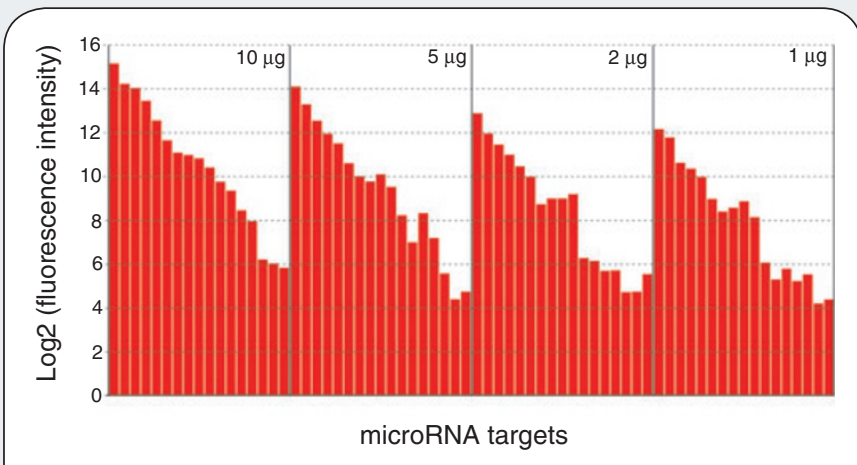

Figure 3 miRCURY LNA Arrays require only $1 \mu \mathrm{g}$ of total RNA to profile miRNA. Identical miRNA profiles were produced from starting amounts of total RNA that span the range from $10 \mu \mathrm{g}$ to $1 \mu \mathrm{g}$, without miRNA enrichment Represented are 17 different miRNAs detected in human lung total RNA (Ambion). Numbers in the top right hand corner of each box show the amount of total RNA used to produce each profile.

step from a total RNA sample to remove larger RNAs and to concentrate the pool of miRNA. Unfortunately, miRNA enrichment wastes the sample and is a potential source of experimental artifacts. Furthermore, methods for amplifying signal from miRNA pools also involve multiple steps, and are thus time-consuming and have potential for introducing error.

Based on preliminary data showing that LNA-based capture probes were highly sensitive for miRNA (to at least 50 attomoles), we developed a 90-min, straightforward protocol for labeling small amounts of RNA (tested down to $1 \mu \mathrm{g}$ ), without the need for miRNA enrichment. We found that the miRNA profile from a $1-\mu \mathrm{g}$ sample of total RNA is virtually identical to that produced from $10 \mu \mathrm{g}$ of total RNA (Fig. 3).

\section{Conclusion}

The combination of highly sensitive LNA-based arrays with a robust and uniform labeling method means that as little as $1 \mu \mathrm{g}$ of sample can be used to obtain an accurate miRNA profile from a biological sample. We believe that this, in combination with reduced sample handling steps, high sensitivity and excellent mismatch discrimination make miRCURY LNA Arrays a substantial advance in miRNA profiling.

1. Castoldi, M. et al. A sensitive array for microRNA expression profiling (miChip) based on locked nucleic acids (LNA). RNA (in the press).

This article was submitted to Nature Methods by a commercial organization and has not been peer reviewed. Nature Methods takes no responsibility for the accuracy or otherwise of the information provided. 